\title{
The Application of Flat Animation in the Field of Communication
}

\author{
Luzhu Zhang \\ Huanggang Normal University \\ Academy of Fine Arts Animation Department Teacher \\ Huanggang, China
}

\begin{abstract}
With the widely used and generally improve the speed of the Internet, micro animation combined with the client terminal device spontaneously reproduced, flat design has injected new vitality into the animation market, it does not need the big screen, no lengthy and complicated plot, through simple shape and micro animation, is a kind of information transfer encoding symbols, information transmission and give the viewer a colorful visual feast. This paper discusses the application of flat animation in the field of communication in the network, in order to promote the form of animation background in the field of communication development.
\end{abstract}

Keywords—flat; micro animation; information; dissemination

\section{INTRODUCTION}

In China, with the development of network technology and application, mobile phone mobile terminal personnel quantity rapid growth, the use of mobile client to browse and transfer information and gradually replace the traditional means of communication, according to the CNNIC statistics report, December 2014, China has 649 million Internet users, mobile phone users reached 557 million, China has built the world's largest 4G network the number of base stations, the size of more than 2 million, the world's first Internet and mobile Internet users, by the end of 2015, China has 688 million Internet users, Internet penetration rate reached $50.3 \%$, in spite of this, there are Chinese Nearly half of the population have become Internet users.

In order to shake the realistic visual effects, I believe everyone will choose to use the theater mode, and flat animation without big screen, in relation to the spread and propagation process of the majority, the interaction can form a mutual adjustment, fine adjustment and joint acquisition, transfer and dissemination of knowledge and civilization in human civilization and rapid development. The spread of today, the art of animation as an art form of the meaning of existence in his transfer of information and knowledge, the traditional media reading and communication apparently has been unable to meet the cultural knowledge of the fast receiving and send demand, more and more people focus on micro-blog, WeChat, APP application in writing Write, forwarding and comments above, this does not need to consume much traffic can trigger

This work is the humanities and social science research youth project of Hubei Provincial Department of Education. The name of the project is flat animation for the integration of audio and video communication - Taking Yiduiread Animation as an Example (item number: 17Q187). the same topic and heat to every grassroots has interactive influence in the mobile client communication experience in knowledge is king of the era.

The rapid spread of the important mass level, in its adoption and use, is a very important factor. The city fast-paced life, the audience can use mobile phone client to spend 1-3 minutes watching animation content a complete theme, it is with a simple design and a deeper impression on it by cutting out the superfluous people. The formation of positive interaction in the process of communication, is a fast-paced life in a stream, a short, flat, fast pace with modern life.

\section{FLAT ANIMATION INFORMATION DISSEMINATION CHARACTERISTICS}

According to the analysis of a number of 20-30 users were stars, most of the young readers can accept the flat design, it makes the network more convenient, can highlight the theme, improve reading efficiency, simple interaction, eliminating redundant functions, easy access to information, is advantageous in the short period of time to understand the full range of information, so flat design is the information media age to express ideas in a form of animation is very important

Flat animation is the dissemination of information in the form of graphic interpretation, its subject use more public concern to people's livelihood, hot news, news and other topics, flexible form, through the interactive mode is simple, is open to the public network platform, click to rate at the same time a large amount of forwarding, each person is connected to the network and the public the cross platform transfer and communication, traditional animation is the traditional China dish is very delicious, to use a fixed broadcast platform to reach the audience there, digestion and absorption and spread it all need, this is obviously slow.

Flat animation theme, modeling simple, almost no hero, with simple geometry on the flat color, then use the combination of music and picture form, increase the fine animation effects, mainly is the vision and hearing synchronous combination, let the information transfer rapidly in a short time, and absorbed by the audience, then quickly transferred to the connection mobile phone media port every point of the hands of the people, only need a forwarding or comments can be made to share the topic, so as to spread like a virus. Flat animation is an important means of information age of the 
Internet makes information transfer rapidly spread, which is the traditional animation can't be achieved.

\section{The AUdiO-VISUAL FEATURES OF FlAt ANIMATION}

\section{A. A Very Simplified Styling Design}

Flat animation information to facilitate the reception and release of the picture form, simple and convenient design makes the information video content with strong operability and can be spread in the age of information explosion. The origin of flat animation dates back to the Second World War, the period of internationalism. In the early days of Mies Van de Lo put forward "less is more" principle 1 Swiss internationalism graphic design, the pursuit of geometric rigor, concise and lively layout, the use of decorative lines without lines, complete shape, thus forming a highly functional and rational design style. The flat shape is emphasized and the proportion of segmentation, that shape itself, with bright colors, in shape design, mostly through geometric based shape such as square, round or triangular, wavy, straight line shape changes.

The flat figure model design as an example, flat face shape, mostly designed without edge shape, just modify the collocation proportion, hairstyle, clothing, decoration, color color as the main color pattern, simple changes can distinguish between male and female, the elderly, children, so as to adapt to various changes, according to the facial features circle, point or line to divide the face space, convenient to do animation, limbs design into strips, no joints, such as shadow, sometimes half shadow half highlighted in the form of design, eliminating the design details, which is different from the traditional skeuomorph design. The characters are generally in a positive image such as the character to the left To right or right to left side of the lens is the image, such as a movie, occasionally showing the person to the $3 / 4$ side, and less use other side view image, so to reduce the action of making unnecessary trouble. In order not to specifically highlight the role of personality or emotion objective set, the role of many tall in proportion, slender limbs, sometimes designed without special gender tendency, this "to" image design, can sometimes be any role on behalf of the public in the image, no occupation age and gender characteristics, on behalf of life in each and every one of you I, meet the popular topic of flat animation for the dissemination of knowledge, it is convenient and fast production Spread.

\section{B. Semi Solid Color Design}

From the color design, color preference dynamic - color is more pure. The main and secondary colors are usually very popular, then with several other colors. No color shading level and rich, collocation gray shadow or long projection, but not empty, the edge color is clear and clean, the brightness changes with little or no color is the function of filling and zoning.

In addition, flat design from the color collocation of speaking, light orange, purple, green, and blue color is also very popular retro. Compared with the traditional animation color, micro animation is more smooth and clean, with grey color combination of produce effect, color and brightness level change is also rich, to meet the needs of the design.

\footnotetext{
${ }^{1}$ Discussion on American Modernism Xupu
}

\section{Audiovisual Consistent Animation Features}

Information transmission has developed for many years. Text language and picture language have gradually formed a unique information transmission coding system. Flat animation integrates the principles of audio-visual consistency in animation, which makes animation performance and text information synchronous and combined, and enhances the multiple transmission mode of information. In professional terms, the synchronization of sound and painting is an important form of any film. Also, simply by combining certain images and specific sounds, the vision and hearing are simultaneously performed 2 .

1) Words in ideographic animation: Usually, the animation combined with text commentary to promote the development of the story, the animation is also changing with the text content of the text, the picture, sound with pictures, text and animation, the two organized by, for example, such as micro EVA creative products company advertising words in "this is a delicious and nutritious food for children and women of ladybugs agent", so the picture appeared in children and women, followed by a variety of delicious food symbols, advertising products intended to recommend a food supplement food for children female agent, when the text "to provide nutritious and delicious food supplement food agent" when the text is More than one, and only one screen picture, they will be a number of food in the same screen, the use of micro animation effects one by one display, slowly presented in front of the audience, in line with the audience's information receiving psychological state. Flat animation is an important medium of dissemination of information, communication is the process of text information and animation combined with the performance, on the one hand it reduces the content in the course of transmission loss, expounds the connotation of information, on the other hand, the information widely and orderly transfer in the terminal equipment, get effective absorption and utilization.

2) Animation to participate in camera transitions: Flat animation in order to ensure the rationality of ornamental fluency and rhythm, the use of animation to switch the lens as transitions, enhances the performance of animation efforts and ornamental, such as in traditional animation scene transitions have fade in, fade, hard switching, voice as transitions, shady transitions, flat animation is the use of graphics rotating zoom, rotation into the narrow, left, right off, jumped into the screen, out of reach to simulate the dynamic effect, push, pull, shake, move, and, throwing shots. For example the lens fade imitation, you can use the same color geometry mosaic, fades collective schedule Is used to switch the camera rotation reduced. Here the geometry including square, circular, polygonal, form a point, line, face animation, animation and visual effects of it and traditional animation is different, it will be micro animation in the "micro" and "small" action to the extreme, with small voice animation, to ensure smooth lens

${ }^{2}$ Combination and application of sound painting in film and television animation Liuming 
below. According to the actual situation, occasionally choose hard cutting performance.

The expression of micro animation than traditional animation rhythm, the so-called micro animation, not only in time, in some micro effect, any objects appear or disappear with micro effect, which in the "one read," said animation animation "UFO" network popular micro video animation performance is particularly evident, the technical term is slow moving effects, scene animation is based on "7-11-15 frame" principle, including Disney animation, many are based on the dynamic principle, taking flash as an example, if the need is for the fifteenth frame effect characters appear in the picture, then began to appear in the display frame seventh this. A character may be larger, 11 frames smaller, and then revert to the desired size in fifteenth frames, which is the jiggle effect.

3) Sound participation in graphic design: The sound and picture in one way audio to the extreme performance in flat animation, a full range of voice performance following the animation, when the sound scene is just the sound performance of the content of the scene, the appearance effect also happens to have the corresponding audio commentary. Often appear in the form of voice, no dialogue and monologue. And always carry out the third aspect, enhance the objectivity and fairness of the impression.

In the case of micro Chinese animation as a main part of the content, and the picture closely relationship, it can participate in the animation, become a part of the picture, animation design of micro parts of the text, the picture is clean and neat, just perfect show content. Text with an objective statement makes an explanation with the viewfinder lens, using panoramic view. With a few, near the scene, close-up involves less subjective emotion, intended to be more objective about panoramic view of things, the characters and characters of panoramic view of the heart and emotions, the service scene content.

\section{Flat ANIMATION HAS A WidE RANGE OF} APPLICATIONS

The Internet has changed to television, radio, newspapers and magazines and cultural knowledge dissemination way, mainly micro-blog, WeChat, APP and other open development platform, to increase the amount of information they receive, the fast pace of life in the flat animation is widely applied, it will text message effectively transfer to the user, the customer also make quick response according to the information received, some text and pictures of the rhythm and animation effects, strengthening the interaction factors of knowledge dissemination, the flattening of the APP animation in the network, network advertising, social networking, network television, network broadcast used up.

In the webcast or interactive, flat animation generally used in network interaction, in the late micro courses to webcast live courses as an example, the early titles is to micro animation to import courses and create a classroom atmosphere, through the micro animation brief open field, mobilize the live emotion in the course the mid micro animation expression with the teacher's teaching, but also conducive to strengthening the interaction between the teacher and the students, through all the time in class, finally, at the end of the course, combined with proper ending animation, forming a complete set of classes.

\section{CONCLUSION}

Micro animation in the Internet era, the diversification of the story to the audience as the main explanation, very rhythmic collocation, design simple and elegant scenes and characters, features a large spread in a short period of time, so that people can watch in their spare time or sneak in, and learn some knowledge, knowledge of visual books and the electronic and network interactive platform function, strengthen the education of knowledge dissemination in the network era of power, as the saying goes: three of us are walking together, there must be my teacher, knowledge from books and textbooks, classroom and teachers are relative. In addition to the dissemination of knowledge and culture, we should pay attention to the reduction in Internet the process of learning Net negative energy transmission, correctly treat various ways, various levels of knowledge and culture, through a variety of channels to learn and disseminate useful content!

\section{REFERENCES}

[1] Xu Yibing Xu Xiao Xiao. Dynamic graphic design [M]. Shanghai: Shanghai people's Fine Arts Publishing House.2013..J. Clerk Maxwell, A Treatise on Electricity and Magnetism, 3rd ed., vol. 2. Oxford: Clarendon, 1892, pp.68-73.

[2] Marcel Maartin. Film language [M]. Beijing. Chinese Film Publishing House,.1980K. Elissa, "Title of paper if known," unpublished.

[3] NIE to. Animation clip [M]. Shanghai. Shanghai Fine Arts Publishing House.2006Y. Yorozu, M. Hirano, K. Oka, and Y. Tagawa, "Electron spectroscopy studies on magneto-optical media and plastic substrate interface," IEEE Transl. J. Magn. Japan, vol. 2, pp. 740-741, August 1987 [Digests 9th Annual Conf. Magnetics Japan, p. 301, 1982].

[4] Xue Yanping. Non mainstream animated films [M]. Beijing. 